\title{
Early tumor shrinkage as a predictor of favorable outcomes in patients with advanced pancreatic cancer treated with FOLFIRINOX
}

\author{
Yasuhiro Kaga ${ }^{1, *}$, Yu Sunakawa ${ }^{1, *}$, Yutaro Kubota ${ }^{2}$, Teppei Tagawa ${ }^{2}$, Taikan \\ Yamamoto $^{1}$, Toshikazu Ikusue ${ }^{1}$, Yu Uto ${ }^{1}$, Kouichirou Miyashita ${ }^{1}$, Hirokazu \\ Toshima $^{3}$, Kouji Kobayashi ${ }^{3}$, Atsushi Hisamatsu ${ }^{3}$, Wataru Ichikawa ${ }^{4}$, Takashi \\ Sekikawa ${ }^{4}$, Ken Shimada ${ }^{3}$, Yasutsuna Sasaki ${ }^{2}$ \\ ${ }^{1}$ Divison of Medical Oncology, Showa University Northern Yokohama Hospital, Yokohama, Japan \\ ${ }^{2}$ Division of Medical Oncology, Showa University School of Medicine, Tokyo, Japan \\ ${ }^{3}$ Divison of Medical Oncology, Showa University Koto Toyosu Hospital, Tokyo, Japan \\ ${ }^{4}$ Division of Medical Oncology, Showa University Fujigaoka Hospital, Yokohama, Japan \\ *These authors contributed equally to this work
}

Correspondence to: Yu Sunakawa, email: y.suna0825@gmail.com

Keywords: pancreatic cancer, FOLFIRINOX, early tumor shrinkage

Received: July 10, $2016 \quad$ Accepted: September 06, 2016

Published: September 13, 2016

\section{ABSTRACT}

There are several reports on the correlation between early tumor shrinkage (ETS) or depth of response (DpR) and survival in chemotherapies for colorectal cancer; however, few studies have investigated it in pancreatic cancer. We therefore investigated whether the ETS will predict outcomes in $\mathbf{5 9}$ patients with advanced pancreatic cancer treated with FOLFIRINOX therapy. The association of ETS with progression-free survival (PFS) and overall survival (OS) was evaluated but also we addressed to the correlation between outcomes and DpR. ETS was defined as a reduction $\geq 20 \%$ of target lesions' diameters measured at 6 to 8 weeks from treatment start. DpR was percentage of maximal tumor shrinkage observed at the nadir diameter compared with baseline. Among 47 evaluable patients for the ETS, $12(25.5 \%)$ patients experienced ETS. The ETS was significantly associated with better PFS (9.0 vs. 4.2 months) as well as OS (24.0 vs. 9.1 months); moreover, the association had a statistically significance for PFS but a strong trend for OS in multivariate analysis. The DpR was statistically significantly but weakly associated with OS. In conclusion, this is the first report that the early response to chemotherapy may predict favorable outcomes in patients with advanced pancreatic cancer treated with FOLFIRINOX therapy.

\section{INTRODUCTION}

Pancreatic cancer is the fourth leading cause of death from cancer over the world, which is usually a high grade of malignancy at the diagnosis and is one of intractable cancers [1]. It carries a severe prognosis, which 5-year survival rate was reported to be lower than $5 \%$ [1]. In addition, the morbidity of the disease is increasing in recent year; therefore, more than 30,000 people die of pancreatic cancer every year in Japan [2]. Advanced pancreatic cancer remains an incurable disease with few cure treatments. The phase III PRODIGE 4/ACCORD 11 trial provided one of current standard regimens for advanced pancreatic cancer, consisting of oxaliplatin, irinotecan, fluorouracil, and leucovorin (FOLFIRINOX), which has superior response rate (RR) and survival benefit even with severe toxicity in patients with advanced pancreatic cancer. The RR, median progression-free survival (PFS), and overall survival (OS) were significantly better in patients receiving FOLFIRINOX therapy compared to patients receiving gemcitabine therapy (RR, 31.6\% vs. 9.4\%; median PFS, 6.4 months vs. 3.3 months, HR 0.47, 95\% CI $0.37-0.59, P<0.001$; median OS, 11.1 months vs. 6.8 months, HR $0.57,95 \%$ CI $0.45-0.73, P<0.001$ ) 
[3]. Since then, FOLFIRINOX regimen has been used as a standard first-line treatment for patients with advanced pancreatic cancer; however, there are still no biomarkers to predict favorable outcomes of the FOLFIRINOX. There have been several reports on the correlation between early tumor shrinkage (ETS) or depth of response (DpR) and survival time in chemotherapies for metastatic colorectal cancer [4-7]. The phase III TRIBE trial compared firstline FOLFOXIRI plus bevacizumab with FOLFIRI plus bevacizumab for unresectable metastatic colorectal cancer patients. In a subgroup analysis of the TRIBE trial, a highly significant correlation of both ETS and DpR with survival parameters (PFS, post progression survival, and OS) was reported in the arm of triplet plus bevacizumab treatment [5]. Moreover, a subgroup analysis of the phase III FIRE-3 trial which evaluated first-line FOLFIRI plus cetuximab versus FOLFIRI plus bevacizumab also demonstrated a strong correlation of ETS with PFS and OS [8]. Recently, Heinemann V et al. reviewed the ETS and DpR for colorectal cancer treatment and evaluated their potential as predictive markers for the clinical management. The review showed that the ETS not only relates to a prognostic category but also distinguishes patients with high sensitivity to treatment and more favorable prognosis from a heterogeneous group of patients classified as no ETS [4]. An observational cohort study for advanced pancreatic cancer patients treated with the triplet regimen has revealed Eastern Cooperative Oncology Group (ECOG) performance status (PS), liver metastases, and neutrophil-to-lymphocyte ratio as the most important predictors of survival [9]. However, to the best of our knowledge, there have been few studies to investigate the association between ETS or DpR and clinical outcomes of chemotherapy in pancreatic cancer. We therefore investigated whether the ETS will predict outcomes in patients with advanced pancreatic cancer treated with FOLFIRINOX therapy.

\section{RESULTS}

\section{Patient characteristics}

Between November 2012 and November 2015, 59 patients were enrolled from 3 institutions of Showa University (Showa University Northern Yokohama Hospital, Showa University Hospital, and Showa University Koto Toyosu Hospital). The patient characteristics at baseline are shown in Table 1. The median age was 63 years (range, 34-76), forty-four patients $(74.6 \%)$ had a PS of 0 . The primary site of tumor was the head of pancreas in 26 patients (44.1\%). Thirteen patients $(22.0 \%)$ had a biliary stent, and 47 patients $(79.7 \%)$ had metastatic diseases. Forty patients $(67.8 \%)$ were treated with FOLFIRINOX as a first-line treatment. In the all population, RR, median PFS, and OS were $27.3 \%, 5.3$ months (95\% CI, 4.2-7.9), and 10.3 months (95\% CI, 7.6-11.1), respectively.

\section{Association between response to the treatment and clinical outcomes}

Among all enrolled patients, 4 patients were not evaluable for response due to patients with locally advanced diseases or peritoneal diseases. Among 55 evaluable patients for response, $15(27.3 \%)$ patients achieved a partial response. The OS was statistically significantly longer in responders compared to nonresponders (24.0 months vs. 9.0 months, HR $0.33,95 \% \mathrm{CI}$ $0.12-0.75, \log$-rank $P=0.010$ ). No significant association was observed between response and PFS (Table 2).

Forty-seven (79.6\%) of all 59 patients had measurable tumors for the ETS. Among the evaluable patients for the ETS, $12(25.5 \%)$ patients experienced an ETS. The PFS was statistically significantly longer in patients with ETS compared to those with no ETS (9.0 months vs. 4.2 months, HR 0.43, 95\% CI 0.18-0.91, log-rank $P=0.032$ ). Moreover, patients with ETS had a significantly better OS (24.0 months vs 9.1 months, HR 0.36, 95\% CI 0.12-0.87, $\log$-rank $P=0.031)($ Table 2, Figure 1).

\section{Association between DpR and clinical outcomes}

Thirty-one patients $(52.5 \%)$ who experienced an OS event were estimated for the DpR. Median DpR was $-10.3 \%$ (from -75.7 to 100 ). The DpR was statistically significantly but weakly associated with OS $\left(r_{\mathrm{s}}=0.18\right.$, $P=0.017$ ) and was not associated with PFS (Figure 2).

\section{Multivariate analysis}

We performed a multivariate analysis for several variables including response and ETS to precisely evaluate the association with clinical outcomes. In the multivariate analysis adjusted for ECOG PS and diagnosis, the response was statistically significantly associated with both PFS and OS (HR 0.38 ; 95\% CI $0.18-0.80 ; P=0.011$, HR $0.33 ; 95 \%$ CI $0.13-0.79 ; P=0.013$, respectively). On the contrary, the ETS had a significant association with PFS $(P=0.02)$ but a strong trend with $\mathrm{OS}(P=0.065)$ (Table 3$)$.

\section{DISCUSSION}

We analyzed the associations between ETS or DpR and clinical outcomes of FOLFIRINOX treatment in patients with advanced pancreatic cancer. To the best of our knowledge, the present analysis is the first attempt to evaluate the association of response to the intensive chemotherapy with survival parameters in pancreatic cancer. Our data showed that response had a significant correlation with OS in the assessable population. Moreover, the ETS was significantly associated with both PFS and OS, suggesting that achieving rapid and deeper response may lead not only to delay of disease progression but also to prolonged survival. 
Table 1: Demographic and baseline characteristics of patients $(n=59)$

\begin{tabular}{lc}
\hline Characteristics & \\
\hline Age-year & 63 \\
Median & $34-76$ \\
Range & \\
Sex-no.(\%) & $40(67.8)$ \\
Male & $19(32.2)$ \\
Female & \\
ECOG PS-no.(\%) & $44(74.6)$ \\
0 & $15(25.4)$ \\
1 & \\
Diagnosis-no (\%) & $12(20.3)$ \\
Locally advanced & $47(79.7)$ \\
Metastatic & 38 \\
Synchronous & 9 \\
Metachronous & \\
Treatment line-no (\%) & $40(67.8)$ \\
First-line & $19(32.2)$ \\
Second-line & \\
Pancreatic tumor location-no.(\%) & $26(44.1)$ \\
Head & $22(37.3)$ \\
Body & $9(15.3)$ \\
Tail & $2(3.4)$ \\
Multicentric & \\
Biliary Stent-no.(\%) & $13(22.0)$ \\
Yes & $46(78.0)$ \\
No &
\end{tabular}

Table 2: Associations of ETS and response with clinical outcomes

\begin{tabular}{ccccc}
\hline & ETS $+(\boldsymbol{n}=\mathbf{1 2})$ & ETS- $(\boldsymbol{n}=\mathbf{3 5})$ & HR $(\mathbf{9 5} \% \mathbf{C I})$ & $\boldsymbol{P}$-value \\
\hline PFS & 9.0 months & 4.2 months & $0.43(0.18-0.91)$ & 0.032 \\
OS & 24.0 months & 9.1 months & $0.36(0.12-0.87)$ & 0.031 \\
\hline & Responder $(\boldsymbol{n}=\mathbf{1 5})$ & Non-responder $(\boldsymbol{n}=\mathbf{4 0})$ & HR $\mathbf{( 9 5 \%}$ CI $)$ & $\boldsymbol{P}$-value \\
\hline PFS & 5.7 months & 4.2 months & $0.56(0.27-1.07)$ & 0.090 \\
OS & 24.0 months & 9.0 months & $0.33(0.12-0.75)$ & 0.010 \\
\hline
\end{tabular}

ETS, early tumor shrinkage; PFS, progression-free survival; OS, overall survival.

FOLFIRINOX is now considered to be one of first-line standard treatments for patients with advanced pancreatic cancer according to results of the phase III trial by Conroy et al. [3]. However, it cannot be applied to all patients with pancreatic cancer due to severe toxicity of the FOLFIRINOX, such as neutropenia, leucopenia, febrile neutropenia, anorexia, and diarrhea. The study by Metges et al. who are the original investigators in the
PRODIGE 4/ACCORD 11 trial, based on their established criteria, showed that $81 \%$ of 242 patients required a dose reduction (median dose intensity; 5-FU bolus $82 \%$, oxaliplatin $78 \%$, irinotecan $81 \%$ ), but that this result did not affect treatment efficacy compared to the PRODIGE 4/ACCORD 11 trial: RR $39 \%$ vs. $32 \%$, median PFS 6.5 vs. 6.4 months, and median OS 10.9 vs. 11.1 months [10]. A Japanese phase II trial of the FOLFIRINOX showed 
that grade 3 or 4 neutropenia and febrile neutropenia were more frequently observed compared to those in the global phase III trial $(77.8 \%$ vs. $45.7 \%, 22.2 \%$ vs. $5.4 \%$, respectively) [11]. Recently, several clinical studies of a modified FOLFIRINOX regimen have been carried out to reduce its toxicities. Blazer et al. reported their experience with reducing irinotecan in addition to dropping 5-FU bolus, and also concluded that the modified regimen was effective and well tolerated with no episodes of grade 3 or 4 neutropenia and thrombocytopenia, but with $46 \%$ of patients requiring a dose reduction for other toxicities [12]. Another Japanese phase II trial which evaluated the efficacy and safety of modified FOLFIRINOX regimen with intravenous oxaliplatin $85 \mathrm{mg} / \mathrm{m}^{2}$, irinotecan
$150 \mathrm{mg} / \mathrm{m}^{2}, 5-\mathrm{FU}$ infusion $2400 \mathrm{mg} / \mathrm{m}^{2}$ over 46 hours, and no bolus 5-FU, demonstrated that the modified regimen has an improved safety profile with maintained efficacy in advanced pancreatic cancer [13]. We therefore clinically utilized irinotecan dosage of $150 \mathrm{mg} / \mathrm{m}^{2}$ for patients enrolled in this study. The survival time (median PFS, 5.3 months; median OS, 10.3 months) in our cohort was similar to that of the Japanese phase II trial of FOLFIRINOX (median PFS, 5.6 months; median OS 10.7 months) as well as the FOLFIRINOX phase III trial (median PFS, 6.4 months; median OS, 11.1 months) [11].

In addition to considering more proper dosage, it would be important to investigate novel predictors for efficacy and survival outcomes of the FOLFOIRINOX
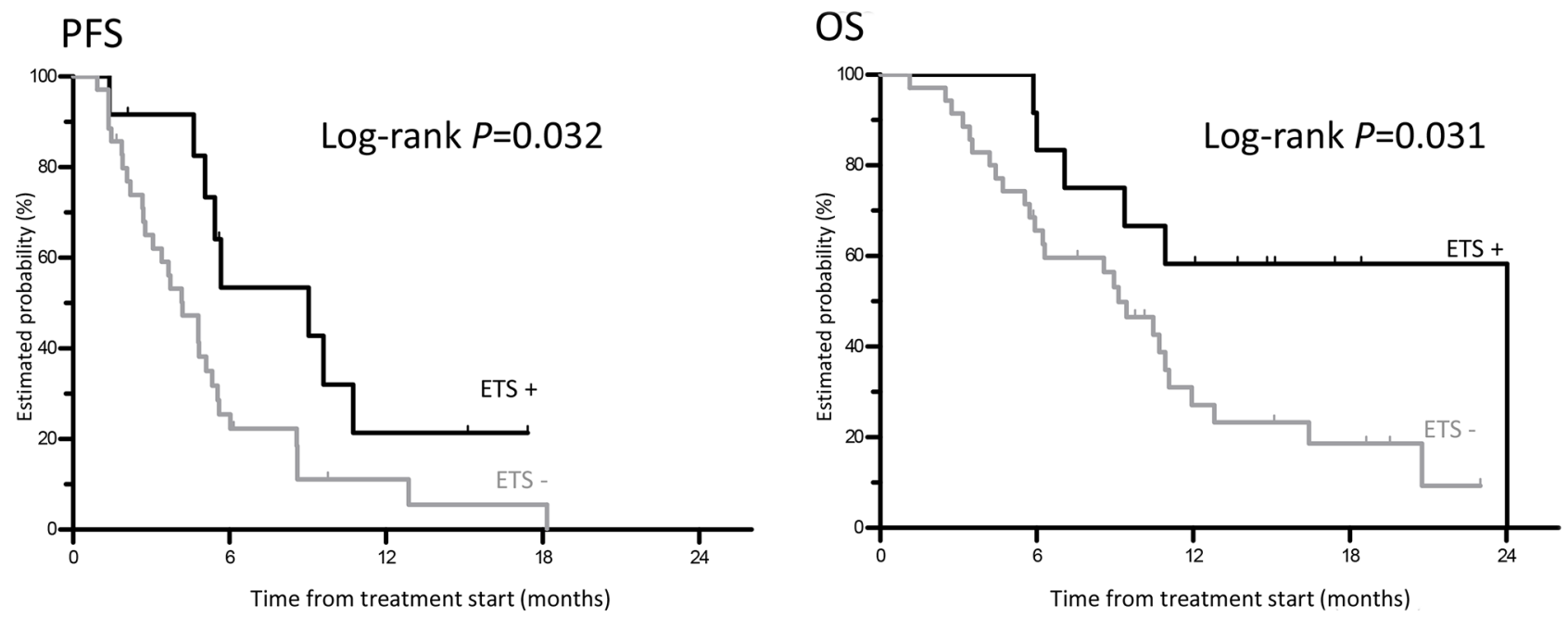

Figure 1: Kaplan-Meier curves for progression-free survival and overall survival in relation to early tumor shrinkage.
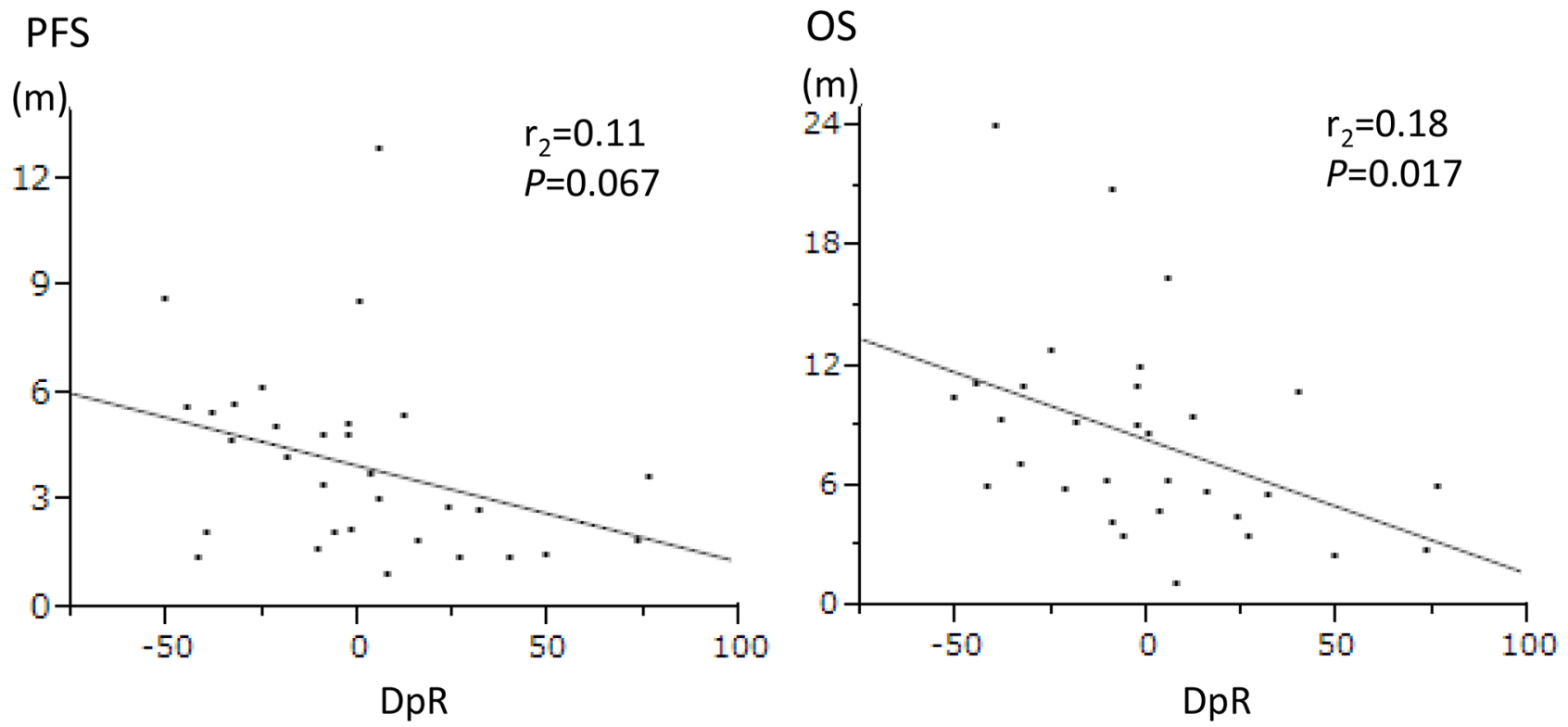

Figure 2: The correlation of depth of response (DpR) with clinical outcomes by the Spearman's rank correlation coefficient. 
Table 3: Multivariate analysis

\begin{tabular}{|c|c|c|c|c|c|c|}
\hline \multirow[b]{2}{*}{ Variables } & \multicolumn{3}{|c|}{ Progression-free survival } & \multirow[b]{2}{*}{ HR } & \multicolumn{2}{|c|}{ Overall survival } \\
\hline & HR & $95 \% \mathrm{CI}$ & $P$-value* & & $95 \% \mathrm{CI}$ & $P$-value* \\
\hline \multicolumn{7}{|l|}{ Gender } \\
\hline Male vs. Female & 1.06 & $0.56-2.00$ & 0.87 & 0.93 & $0.48-1.81$ & 0.82 \\
\hline \multicolumn{7}{|l|}{ ECOG PS } \\
\hline 0 vs. 1 & 0.56 & $0.29-1.09$ & 0.086 & 0.45 & $0.22-0.91$ & 0.026 \\
\hline \multicolumn{7}{|l|}{ Diagnosis } \\
\hline Locally advanced vs. metastatic & 0.32 & $0.12-0.84$ & 0.021 & 0.33 & $0.11-0.94$ & 0.037 \\
\hline \multicolumn{7}{|l|}{ Response } \\
\hline Responder vs. non-responder & 0.38 & $0.18-0.80$ & 0.011 & 0.33 & $0.13-0.79$ & 0.013 \\
\hline \multicolumn{7}{|l|}{ ETS } \\
\hline ETS+ vs. ETS- & 0.37 & $0.16-0.85$ & 0.020 & 0.40 & $0.15-1.06$ & 0.065 \\
\hline
\end{tabular}

treatment in pancreatic cancer. The predictive factor would enable physicians to distinguish patients who benefit more from the intensive chemotherapy with potentially severe toxicities. A subgroup analysis of the TRIBE trial evaluating a triplet regimen plus bevacizumab for colorectal cancer has reported ETS and DpR as promising surrogates for survival time [5]. The ETS, which is decrease in tumor load measured at the time of first imaging after the start of treatment, is considered to be an early indicator of sensitivity to chemotherapy for metastatic colorectal cancer. The $\mathrm{DpR}$ is defined as the percentage of tumor shrinkage, based on the longest diameters or reconstructed volume, observed at the lowest point, so called nadir, compared with baseline [4]. FOLFOXIRI plus bevacizumab improved the ETS and DpR when compared with FOLFIRI plus bevacizumab in a sub-analysis of the TRIBE trial [14]. Moreover, the analysis showed achieving rapid and deep tumor shrinkage consistently delayed tumor progression and prolonged survival in patients treated with the triplet-based regimen. Therefore, the ETS and DpR may become novel factors to predict favorable outcomes of FOLFIRINOX treatment in patients with advanced pancreatic cancer.

Our study could demonstrate for the first time that ETS to the intensive chemotherapy with triplet regimen may predict favorable outcomes in patients with advanced pancreatic cancer. The ETS was significantly associated with better PFS as well as OS in patients treated with the FOLFIRINOX therapy; moreover, the association had a statistically significance for PFS but a strong trend for OS in multivariate analysis. The responders had significantly longer OS compared to the non-responders in both univariate and multivariate analyses. In addition, the $\mathrm{DpR}$ was statistically significantly but weakly associated with OS but not PFS. The ETS is an assessment method that is usually judged at earlier timing from treatment start than response to chemotherapy and DpR; therefore, it may enable us to distinguish patients earlier, who benefit more from FOLFIRINOX therapy. Survival time is much shorter in pancreatic cancer compared to colorectal cancer, leading to shorter treatment duration. Thus, it may be reasonable to switch regimen for ETS-negative patients. Our findings and hypothesis need a prospective validation which may contain an adaptive trial design to evaluate treatment decision according to ETS status.

Our study was retrospective, and included a limited assessable population because of small number of patients. FOLFIRIXNOX regimen is usually used as a first-line treatment for advanced pancreatic cancer; therefore, the ETS and DpR should be investigated in patients receiving the triplet regimen as an initial therapy. We performed a sub-analysis according to treatment line; however, we failed to indicate the significant association between the biomarkers and clinical outcomes due to small sample size $(n=31)$ (data not shown). It is desirable to assess them prospectively for achieving more accurate results using larger patient cohorts. In previously reported trials which investigated association of the ETS with clinical outcomes, the tumor assessment was carried out every 8 weeks until the evidence of disease progression by means of computer tomography scan $[4,5,15]$; however, we measured target lesions for assessment of ETS at 6 or 8 weeks from treatment start due to retrospective trial and limited data, causing a difference in the timing of evaluation for ETS from previous reports. We therefore should integrate the term of evaluation for ETS in future studies.

In conclusion, the ETS and DpR were significantly associated with clinical outcomes in advanced pancreatic cancer patients treated with FOLFIRINOX chemotherapy. Our results also suggest that the early response to the triplet regimen may predict better clinical outcomes in patients with advanced pancreatic cancer. These findings are warranted to validate in future trials. 


\section{MATERIALS AND METHODS}

\section{Patient}

This study enrolled advanced pancreatic cancer patients with an ECOG PS of 0 or 1, and adequate hematological, liver, and renal function, who received FOLFIRINOX as first- or second-line treatment between November 2012 and November 2015 in 3 institutions of Showa University (Showa University Northern Yokohama Hospital, Showa University Hospital, and Showa University Koto Toyosu Hospital). All clinical data were obtained retrospectively from their medical records.

\section{Treatment}

We utilized the FOLFIRINOX with modified dosage according to the Japanese phase II trial [13]: irinotecan was also infused intravenous (i.v.) over 90 minutes at $150 \mathrm{mg} / \mathrm{m}^{2}$ and then 2 hours i.v. infusion of oxaliplatin at $85 \mathrm{mg} / \mathrm{m}^{2}$ and 2 hours i.v. infusion of 1-leucovorin at $200 \mathrm{mg} / \mathrm{m}^{2}$ followed by continuous i.v. infusion of 5 -FU over 46 hours at $2400 \mathrm{mg} / \mathrm{m}^{2}$. This regimen was repeated every two weeks until disease progression. Dose modifications were made at the treating physician's discretion. Patient received palonosetron, aprepitant, and dexamethasone for emesis prophylaxis.

\section{Study design}

We retrospectively analyzed characteristics, RR, PFS, and OS in patients enrolled in this study. The objectives of our study were to evaluate the association of ETS with PFS and OS but also to address the correlation between clinical outcomes and DpR. Responses were evaluated according to the RECIST version 1.1 by the investigators. ETS was defined as a reduction $\geq 20 \%$ of target lesions' diameters measured at 6 to 8 weeks from treatment start. DpR was defined as the percentage of maximal tumor shrinkage observed at the nadir diameter compared with baseline. A DpR of negative 100\% indicates the complete disappearance of all target tumor lesions. We thought it was difficult to take an accurate measurement of the primary site of tumor in patients with locally advanced pancreatic cancer. Then, we mainly evaluated metastatic measurable lesions in patients with metastatic pancreatic cancer for evaluation of ETS and DpR.

\section{Statistical analysis}

Kaplan-Meier analysis was used to generate survival curves of PFS and OS. The median survival time was compared using the log-rank test. The Spearman's rank correlation coefficient was adopted to evaluate the association between DpR and survival including PFS and
OS. Multivariate analysis was performed based on Wald test for PFS and OS in the multivariable Cox regression model adjusted for ECOG PS (0 vs. 1) and diagnosis (locally advanced vs. metastatic).

Statistical analyses were carried out using JMP 9.0.3 (SAS institute, Cary, NC, USA).

\section{ACKNOWLEDGMENTS}

We thank the patients, their families, and the investigators who participated in this study. We also thank Sachika Koyama for editorial assistance.

\section{CONFLICTS OF INTEREST}

The authors disclose no potential conflicts of interest.

\section{FUNDING AND GRANT SUPPORT}

None.

\section{REFERENCES}

1. Siegel R, Naishadham D, Jemal A. Cancer statistics, 2012. CA Cancer J Clin. 2012; 62:10-29. doi: 10.3322/caac.20138.

2. Center for Cancer Control and Information Services NCC, Japan. http://www.ncc.go.jp/en/cis/index.html.

3. Conroy T, Desseigne F, Ychou M, Bouche O, Guimbaud R, Becouarn Y, Adenis A, Raoul JL, Gourgou-Bourgade S, de la Fouchardiere C, Bennouna J, Bachet JB, KhemissaAkouz $\mathrm{F}$, et al. FOLFIRINOX versus gemcitabine for metastatic pancreatic cancer. N Engl J Med. 2011; 364:1817-1825. doi: 10.1056/NEJMoa1011923.

4. Heinemann V, Stintzing S, Modest DP, Giessen-Jung C, Michl M, Mansmann UR. Early tumour shrinkage (ETS) and depth of response (DpR) in the treatment of patients with metastatic colorectal cancer (mCRC). Eur J Cancer. 2015. doi: 10.1016/j.ejca.2015.06.116.

5. Cremolini C, Loupakis F, Antoniotti C, Lonardi S, Masi G, Salvatore L, Cortesi E, Tomasello G, Spadi R, Zaniboni A, Tonini G, Barone C, Vitello S, et al. Early tumor shrinkage and depth of response predict long-term outcome in metastatic colorectal cancer patients treated with first-line chemotherapy plus bevacizumab: results from phase III TRIBE trial by the Gruppo Oncologico del Nord Ovest. Ann Oncol. 2015. doi: 10.1093/annonc/mdv112.

6. Piessevaux H, Buyse M, Schlichting M, Van Cutsem E, Bokemeyer C, Heeger S, Tejpar S. Use of early tumor shrinkage to predict long-term outcome in metastatic colorectal cancer treated with cetuximab. J Clin Oncol. 2013; 31:3764-3775. doi: 10.1200/JCO.2012.42.8532.

7. Douillard JY, Siena S, Peeters M, Koukakis R, Terwey JH, Tabernero J. Impact of early tumour shrinkage and resection on outcomes in patients with wild-type RAS metastatic 
colorectal cancer. Eur J Cancer. 2015; 51:1231-1242. doi: 10.1016/j.ejca.2015.03.026.

8. Stintzing S, Modest DP, Fischer von Weikersthal L, Decker T, Kiani A, Vehling-Kaiser U, Al-Batran S, Heintges T, Lerchenmueller C, Kahl C, Seipelt G, Kullmann F, Scheithauer W, et al. LBA11 Independent Radiological Evaluation of Objective Response Early Tumor Shrinkage, and Depth Of Response In FIRE-3 (AIO KRK-0306) in the Final RAS Evaluable Population. Annals of Oncology. 2014; 25. doi: 10.1093/annonc/mdu438.9.

9. Vivaldi C, Caparello C, Musettini G, Pasquini G, Catanese S, Fornaro L, Lencioni M, Falcone A, Vasile E. First-line treatment with FOLFOXIRI for advanced pancreatic cancer in clinical practice: Patients' outcome and analysis of prognostic factors. Int J Cancer. 2016; 139:938-945. doi: 10.1002/ijc.30125.

10. Metges JP, Ramee JF, Douillard J-Y, Boucher E, Faroux R, Guerin-Meyer V, Cumin I, Miglianico L, Le Roux C, Dupuis O, Porneuf M, Lam YH, Achour N, et al. Efficacy and safety of FOLFIRINOX in patients with metastatic pancreatic cancer. ASCO Meeting Abstracts. 2014; 32:305.

11. Okusaka T, Ikeda M, Fukutomi A, Ioka T, Furuse J, Ohkawa S, Isayama $\mathrm{H}$, Boku N. Phase II study of FOLFIRINOX for chemotherapy-naive Japanese patients with metastatic pancreatic cancer. Cancer Sci. 2014; 105:1321-1326. doi: 10.1111/cas.12501.

12. Blazer MA, Wu CS-Y, Goldberg RM, Phillips GS, Schmidt CR, Muscarella P, El-Dika SS, Walker JP, Krishna SG, Groce JR, Wuthrick EJ, Williams TM,
Efries D, et al. Tolerability and efficacy of modified FOLFIRINOX (mFOLFIRINOX) in patients with borderline-resectable pancreatic cancer (BRPC) and locally advanced unresectable pancreatic cancer (LAURPC). ASCO Meeting Abstracts. 2014; 32:275.

13. Ueno M, Ozaka M, Ishii H, Sato T, Ikeda M, Uesugi K, Sata N, Miyashita K, Mizuno N, Tsuji K, Okusaka T, Furuse J. Phase II study of modified FOLFIRINOX for chemotherapy-naive patients with metastatic pancreatic cancer. ASCO Meeting Abstracts. 2016; 34:4111.

14. Cremolini C, Loupakis F, Antoniotti C, Lonardi S, Masi G, Salvatore L, Cortesi E, Tomasello G, Spadi R, Zaniboni A, Tonini G, Barone C, Vitello S, et al. Early tumor shrinkage and depth of response predict long-term outcome in metastatic colorectal cancer patients treated with first-line chemotherapy plus bevacizumab: results from phase III TRIBE trial by the Gruppo Oncologico del Nord Ovest. Ann Oncol. 2015; 26:1188-1194. doi: 10.1093/annonc/ mdv112.

15. Tsuji A, Sunakawa Y, Ichikawa W, Nakamura M, Kochi M, Denda T, Yamaguchi T, Shimada K, Takagane A, Tani S, Kotaka M, Kuramochi H, Furushima K, et al. Early Tumor Shrinkage and Depth of Response as Predictors of Favorable Treatment Outcomes in Patients with Metastatic Colorectal Cancer Treated with FOLFOX Plus Cetuximab (JACCRO CC-05). Target Oncol. 2016. doi: 10.1007/ s11523-016-0445-6. 